\title{
Japón: la Era Reiwa ante el déficit de lo social
}

\section{María Elena Romero Ortiz *}

Carlos Uscanga Prieto ${ }^{* *}$

\section{Resumen}

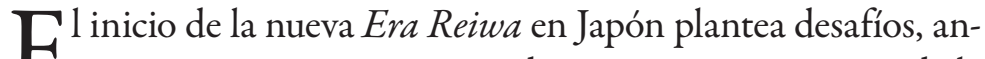
Cte un escenario con ausencia de un mayor crecimiento de la economía, el escalamiento de problemas internacionales que impactan directamente y una sociedad resiliente, pero con crecientes problemas no son desafíos de hoy sino constantes del pasado. El tema social cobra relevancia por su impacto en los ámbitos señalados. Los procesos de envejecimiento se han profundizado, la disparidad en el ingreso, la precariedad laboral, la desesperanza en los jóvenes son características de la sociedad japonesa actual. La transición inédita de la Era Heisei a la Era Reiwa propicia espacios para la reflexión. El presente artículo tiene como objetivo revisar los retos que enfrenta la sociedad japonesa en este nuevo escenario, al centrarse en el análisis de la situación de los jóvenes, al partir del supuesto de que el modelo de desarrollo económico ha generado fracturas profundas en el modelo de bienestar social edificado en la Posguerra, al agravar las desigualdades en el ingreso y el surgimiento de la pobreza relativa en ese país asiático. Asimismo, como fenómeno emergente se ha generado la exclusión de

\footnotetext{
* Doctora en Ciencias Políticas y Sociales con orientación en Relaciones Internacionales por la Universidad Nacional Autónoma de México. Pertenece al Sistema Nacional de Investigadores con el Nivel I y es Profesora-Investigadora de la Facultad de Ciencias Políticas y Sociales de la Universidad de Colima. meromero@ucol.mx

** Doctor en Cooperación Internacional por la Universidad de Nagoya. Pertecene al Sistema Nacional de Investigadores con el Nivel II y es Profesor Titular del Centro de Relaciones Internacionales de la Facultad de Ciencias Políticas y Sociales de la UNAM. auscanga@politicas.unam.mx
} 
jóvenes de padres extranjeros (muchos de ellos nacidos en Japón) que se encuentran ausentes de mayores oportunidades de las que estrictamente se define bajo su calidad migratoria, todos ellos representan a un nuevo grupo: los Dreamers de Japón.

Palabras clave
Era Reiwa, Sociedad, Abenomics, Jóvenes japoneses, Dreamers

Fecha de recepción:

Fecha de aceptación:

Marzo de 2020

junio de 2020

Japan: The Reiwa Era in the Face of the Deficit of the Social

Key words

Reiwa Era, Society, Abenomics, Japanese Youth, Dreamers.

Final submission:

March 2020
Acceptance:

June 2020

\section{Abstract:}

The begining of a new era in Japan, the Reiwa Era face multiple challenges. The absence of further growth of the economy, the escalation of international problems that impact Japan, and a resilient society, but with growing problems, are not today's challenges but constant ones of the past. The social issue becomes a due to its political and economic relevance. Issues such as an aging society, income disparity, job insecurity, hopelessness in young people are characteristics of current Japanese society. The unprecedented transition from the Heisei Era to the Reiwa Era fosters spaces for reflection. So, the objective of this work is to assess the challenges facing Japanese society in this new scenario, focusing on the analysis of the situation of young people, based on the assumption that the model of economic development has generated deep fractures in the Post-war social welfare model, which has been exacerbating income inequalities and the emergence of relative poverty. Likewise, as an emerging phenomenon, the exclusion of young people of foreign parents (many of them born in Japan) who are absent from greater opportunities than those strictly defined by their migratory quality has been generated, all of them represent a new group: the Dreamers from Japan. 


\section{Introducción}

Es un hecho que los últimos treinta años han sido testigos de importantes transformaciones para Japón y para el mundo entero. Es un tiempo complejo que impulsa reformas de todo orden: en lo político, en la economía y lo social. Japón, como la tercera economía del mundo, definió un programa de reestructuración económica en 2012, al inicio del segundo periodo el primer ministro Shinzō Abe. El programa conocido como Abenomics tuvo como fundamento la política de "tres flechas", sustentadas en una agresiva política monetaria, una de orientación fiscal flexible y una estrategia de crecimiento que incluía una reforma estructural. ${ }^{1}$ Sin embargo, una pregunta pertinente en este complejo escenario es, saber si el gobierno, las corporaciones privadas y sociedad japonesa han sido asertivos o no al ajustarse a estos vertiginosos cambios.

El tema social, particularmente cobra relevancia por su impacto en los ámbitos políticos y económicos. Los procesos de envejecimiento se han profundizado, la disparidad en el ingreso es, hoy día - de nuevo- una realidad que marca diferencias antaño ya superadas, las patologías sociales (siempre presentes) florecen. Hay desesperanza e incertidumbre en las nuevas generaciones de jóvenes japoneses en la actualidad. En lo externo, el patrón de hegemonía en el Pacífico ha cambiado ante el meteórico posicionamiento de China. Las amenazas a la seguridad regional escalaron frente a las estrategias de Corea del Norte, mientras las tensiones relacionadas a las reclamaciones fronterizas están presentes y la carrera para mejorar capacidades militares va en aumento en los países de la región. A lo anterior se suma la llamada crisis del multilateralismo, el surgimiento de nuevas expresiones del neopopulismo (de izquierda o de derecha), la crítica a la globalización y al libre comercio; hechos que tan sólo hace un par de años eran inimaginables.

1 Japan Goverment, "ABENOMICS. For future growth, for future generations, and for a future Japan that is robust", (2017). Disponible en https://www.japan.go.jp/abenomics/_userdata/abenomics/pdf/170508_abenomics.pdf 
En el marco de los estudios sobre Japón, surgen preguntas obligadas: ¿Dónde está Japón en ese escenario?, ¿qué hace para estar visible?, ¿qué se espera de ese país como actor en un mundo con grandes turbulencias? Las viejas estrategias usadas por Tokio en la Posguerra ya no son funcionales. La "diplomacia de chequera" es ya historia. El apostar al paraguas de seguridad de los Estados Unidos ya tiene sus limitantes. Japón dejó de ser, en muchos sentidos, el modelo que (como se pregonaba en los años ochenta) podían seguir otras naciones del mundo. La tradicional estructura del sistema político japonés muestra señales de agotamiento. Guillermo Quartucchi observa a un "Japón ensimismado"; Florentino Rodao ve a un "Japón vulnerable y en soledad"; ${ }^{3}$ mientras que Jeff Kingston ${ }^{4}$ percibe a un Japón con nubes en el horizonte. Así, nosotros consideramos a un "Japón carente de fuerza y sentido de dirección”, misma que permitió enfrentar el encuentro con el "llamado Occidente" en el siglo XIX, y la que la hizo resurgir de la devastación después de la Segunda Guerra Mundial.

La Era Hesei llegó a su término para dar paso a la Era Reiwa el 1 de mayo de 2019, marcada por la abdicación del emperador Akihito 5 (ahora Emperador emérito) y el ascenso del nuevo Emperador, Hiro-no-miya Naruhito Shinnō (en adelante Naruhito), lo que significó una transición inédita en Japón y propicia un espacio para la reflexión. En ese sentido, el presente artículo tiene como objetivo realizar una revisión general sobre los retos que enfrenta la sociedad japonesa en este nuevo escenario, centrándose en el análisis de la situación de los jóvenes y su precariedad dentro del universo de los problemas emergentes de la sociedad japonesa contemporánea, identificando en ese contexto a los gru-

5 Su nombre completo es Akibito Tsugu-no-miya. 
pos vulnerables, (conocidos como Freeter, $^{6}{ }^{N E E T s^{7}}$ ) o invisibles como los denominados Dreamers.

Asimismo, se parte de un supuesto central de análisis basado en que el bajo rendimiento del modelo de desarrollo económico en Japón, ${ }^{8}$ iniciado a principios de la década de los noventa con el estallido de la burbúja económica y del cambio del patrón de industrialización, ${ }^{9}$ ha generado fracturas profundas en el modelo de bienestar y en el pacto social edificado en la Posguerra, agravando las desigualdades en el ingreso y el surgimiento de la pobreza relativa en ese país asiático. Las políticas gubernamentales, en lo general, han sido lentas para reaccionar a los cambios que implican el envejecimiento poblacional y los retos que ese proceso conlleva en el ámbito no sólo económico sino también en el social. En ese sentido, el arribo de la nueva Era Reiwa no implica cambios sustanciales en las políticas públicas o de las acciones de Japón en el plano internacional. En lo específico, en los temas de la agenda social, parecería que se mantendría la ausencia de una clara dirección para atender a las emergentes necesidades de las nuevas generaciones de ciudadanos japoneses y de los grupos imperceptibles como los Dreamers.

6 Es un neologismo que viene de los años 80 s y está compuesto por la palabra inglesa free (libre) y la alemana Arbeiter (trabajador). Este término describe la situación de un cierto grupo de jóvenes (entre los 15 y los 34 años) de la sociedad japonesa que, tras terminar sus estudios, eligen trabajar en empleos de medio tiempo, precarios y breves, a menudo dependiendo de sus padres.

7 Es el acrónimo de las siglas en inglés que se refieren a "Not in Employment, Education or Training".

8 Mientras que la tasa anual de crecimiento entre 1951 a 1973 fue de $9.1 \%$, después de el estallido de la burbúja económica a inicios de los años noventa, Japón ha tenido un largo estancamiento de su economía, con periodos recesivos. En 1992, el crecimiento real del Producto Interno Bruto (PIB) fue 1.8\%, una década después fue de $0.9 \%$ para registrar en el 2018 el $0.8 \%$. Véase IMF. "World Economic Outllok Database", International Monetary Forum (2019). Disponible en: https://www. $\mathrm{imf.org/external/pubs/ft/weo/2019/01/weodata/index.aspx.}$

9 Véase Victor López Villafañe y Carlos Uscanga (Coords.). Japón después de ser el Número Uno. Del Alto Crecimiento al Rápido Envejecimiento (Siglo XXI Editores, México, 2015), 232. 


\section{Una mirada a la sociedad japonesa}

La Era Heisei terminó el 30 de abril de 2019. Una serie de rituales en la casa del crisantemo dieron paso a la Era Reiwa a partir del 1 de mayo del mismo año con la llegada del nuevo emperador de Japón: Naruhito. La Era Heisei, a pesar de su significado de "paz universal", fue un periodo en la historia contemporánea de gran turbulencia. En la Era Heisei, Akihito, se convirtió el 8 de enero de 1989 como el primer Emperador en tomar el trono bajo la constitución de la Posguerra de Japón, asumió un papel de "símbolo" del Estado y de la unidad del pueblo japonés. Aunque, la carta magna japonesa le otorga facultades para el nombramiento del Primer Ministro designado por la Dieta japonesa, la promulgación de leyes y tratados, así como otras prerrogativas, lo limita a que "no tendrá poderes relacionados con el gobierno". ${ }^{10}$

Durante la Era Heisei, Japón atravesó una crisis económica prolongada que marcó el comienzo de lo que se llegó a conocer como las décadas perdidas de la economía japonesa. Además, Japón que había sido la segunda economía más grande del mundo después de Estados Unidos, fue superado por China en 2010. ${ }^{11}$ Asimismo, golpeada por grandes desastres naturales siendo uno de los más graves el terremoto y maremoto de la región de Tōhoku del 11 de marzo de 2011, cuya recuperación se sumó a otros desafíos estructurales, entre ellos, el rápido envejecimiento de su población y una sociedad joven con expectativas y sin respuestas. Estos retos no desaparecerán con la transición de la Era Heisei a la Era Reiwa, seguirán estando en la lista de pendientes para la administración de Shinzō Abe y para los futuros gobiernos de Japón.

En la política, Japón vivió una serie de cambios, renuncias y periodos muy cortos de gobierno provocados por escándalos, como la del primer ministro, Noboru Takeshita, quien dejó el poder en ju-

\footnotetext{
${ }^{10}$ Véase capítulo I Art. 4 de la constitución japonesa referente al Emperador, en: Japan Goverment. "The Constitution of Japan”. Disponible en https://japan.kantei.go. jp/constitution_and_government_of_japan/constitution_e.html

${ }^{11}$ Linda Sieg. “Japan's Heisei era: Changes, growth and tragedies”. Reuters (24 de abril de 2019). Disponible en https://www.reuters.com/article/us-japan-emperor-era/japans-heisei-era-changes-growth-and-tragedies-idUSKCN1S00LM
} 
nio de 1989 por una denuncia de compra-venta de favores. Después de Takeshita, diecisiete primeros ministros fueron electos (incluido Shinzō Abe dos veces) entre 1989 al 2019. El Partido Liberal Democrático (PLD) perdió en 1993 y recuperó su posición en 1994 a partir de una alianza con los socialistas; después de 15 años volvió a perder frente al Partido Demócrata de Japón (PDJ), partido que se vió golpeado por las cuestionadas respuestas a la crisis que provocó la triple catástrofe (humana, material y nuclear) por el terremoto y maremoto que impactó a la región de Tōhoku en 2011.

En ese contexto, de incertidumbre y de descontento social por el manejo de la crisis por parte del gobierno japonés, el PLD recuperó nuevamente su posición con el segundo mandato de Abe en 2012. En suma, la Era Heisei enfrentó en lo económico, lo político y en lo social graves problemas y desafíos que no fueron cabalmente superados.

\section{La Era Reiwa}

Desde el punto de vista cultural, el cambio de Era tiene un significado especial en la población. Japón es el único país que mantiene un sistema con nombres de Eras imperiales desde el año 645, cuando inició la Era Taika. Después, en la segunda guerra mundial, el sistema perdió legitimidad bajo la nueva constitución, pero continuó siendo usado -en la práctica- al permitir que se mantuviera en el imaginario colectivo de la población y la figura del Emperador diera a la sociedad japonesa un sentido de identidad y cohesión. En 1979, se reinstaló el fundamento legal que le dio sentido nuevamente a los nombres de las Eras imperiales.

La Era Reiwa nombre inspirado en una antología de poesía japonesa. De acuerdo al secretario en jefe del gabinete, Yoshihide Suga, el concepto Reiwa tiene un amplio significado referido a que "la cultura nace y crece en medio de los corazones de las personas que se atraen maravillosamente ${ }^{112}$ y afirma que la adminis-

\footnotetext{
${ }^{12}$ Katsuya Takahashi. "New Japanese era name Reiwa has lifted society: Suga”. Mainichi Shinbum (1 de mayo de 2019). Disponible en: https://mainichi.jp/english/ articles/20190501/p2a/00m/0na/023000c.
} 
tración de Shinzō Abe desea que en esta nueva Era, los niños y la gente adulta puedan vivir en una "generación de seguridad social".

La Era Reiwa inició en un contexto complejo con nuevos partidos políticos (con diferentes corrientes ideológicas) que se han consolidado como opciones para el electorado japonés, pero al mismo tiempo apuestan por la permanencia del PLD. La agenda política internacional e interna se ve marcada por la discusión sobre temas como el replanteamiento de las estrategias de seguridad nacional frente a las emergentes amenazas en el Este de Asia y el proceso de reforma del artículo 9 de la constitución. En el terreno económico y social, la precariedad laboral, la situación de la mujer, los retos del gasto público frente a los cambios demográficos, por mencionar sólo algunos. Es claro que, a pesar de que el representante de la casa imperial ha cambiado, las bases el sistema político japonés continúan inamovibles y el modelo neoliberal sigue su curso. La apuesta por parte de Japón al Abenomics, ${ }^{13}$ se justifica ante la necesidad para atender los cambios y responder a las crecientes necesidades internas y externas en el mantenimiento de un Japón competitivo, sin embargo, la limitaciones de las reformas emprendidas han sido evidentes y ya muestra esa estrategia económica signos de agotamiento.

Después de que el Producto Interno Bruto (PIB) per cápita se ubicó como uno de los más altos en el mundo, al favorecer un boom consumista, en los años noventa se desplomó y con ello propició cambios en la sociedad, no sólo en términos de su capacidad adquisitiva y de sus niveles de bienestar, si no en la imagen de prosperidad que Japón tenía en el mundo. De acuerdo con Keizo Takenaka "el PIB per cápita de Japón, un indicador de la riqueza material de las personas, se ubicó en los niveles más altos del mundo (en términos de paridad de poder adquisitivo) en los primeros años de Heisei, pero ahora se encuentra cerca de vigésimo quinto en el mundo. Su PIB per cápita actual es menos de la mi-

${ }^{13}$ Carlos Uscanga (Coord). Japón y sus Alternativas de Desarrollo Económico hacia el Futuro (México: Seminario Universitario de Estudios Asiáticos-Universidad Nacional Autónoma de México, 2017), 163-168. 
tad de los niveles de Luxemburgo y Noruega, que ocupan las primeras posiciones". ${ }^{14}$

En una perspectiva más amplia, es claro que las decisiones de política interna -así como de política exterior- repercutirán en la sociedad japonesa; por lo que es menester revisar el tema social a partir de la premisa de que Japón se encuentra en una encrucijada. Al hacer una reflexión sobre la capacidad de la sociedad para asumir esos compromisos y retos, se parte de la afirmación de Yoshio Sugimoto $^{15}$ que plantea que acercarse a la sociedad japonesa genera una serie de interrogantes hipotéticas que inspiran la imaginación sociológica.

En efecto, la imagen de un Japón próspero, homogéneo, limpio, desarrollado, organizado, tecnológicamente avanzado es la que prevalece en el mundo; también se vislumbra a un Japón como un arquetipo de desarrollo y avance. Sin embargo, hoy también se observa una creciente polarización social que incentiva movimientos sociales y levanta voces que reclaman visibilidad a sus problemas.

Temas como la lealtad en una sociedad de orientación grupal, y el respeto a las jerarquías que garantiza un ambiente de armonía, se considera que explican el nivel excepcionalmente alto de estabilidad y cohesión en la sociedad japonesa, que ha ayudado a los líderes políticos y otros en sus esfuerzos por organizar o movilizar a la población de manera eficiente, ${ }^{16}$ hoy día esa estructura es cuestionada y propone retos al gobierno y a la sociedad misma.

Ahora se aprecia a una sociedad más participativa y comprometida para unirse a otros sectores sociales en acciones que permitan encontrar la solución a temas que atañen a todos, como el uso seguro de la energía nuclear, la visibilización de la mujer, la atención a los adultos mayores, el problema del aislamiento de los jóvenes, entre otros temas.

\footnotetext{
${ }^{14}$ Keizo Takenaka. "Meeting the Reiwa Era's challenges". The Japan Times (28 de abril de 2019). Disponible en: https://www.japantimes.co.jp/opinion/2019/04/28/ commentary/japan-commentary/meeting-reiwa-eras-challenges/\#.XRE6Xz9KgdV

15 Yoshio Sugimoto, An Introduction to Japanese Society (London: Cambridge University Press, 2010), 1.
}

${ }^{16}$ Ibidem. 
Dentro de los modestos índices de crecimiento de la economía japonesa, las empresas tienen las prerrogativas de contratar y despedir a los empleados que les permitan una actividad competitiva, favoreciendo la contratación de trabajadores por proyecto o de tiempo parcial. Asimismo, no existe la igualdad laboral para las mujeres y el aumento de espacios para ellas en lugares de alto mando están limitados a pesar de la ley de igualdad de oportunidades para el empleo. ${ }^{17}$ Esa disparidad de género es un reto a superar. Japón ocupa el lugar 110 en la brecha de género global del Foro Económico Mundial.

De la mano del empleo, se encuentra el tema del envejecimiento de la población. Dos cuestiones se derivan de ese problema. Por un lado, la brecha generacional limita la población económicamente activa (PEA), es decir, la mano de obra capacitada para sacar adelante a la economía japonesa se agota, la disminución de nacimientos significará indudablemente en el corto plazo un reto económico. ${ }^{18}$ Por otro lado, el gasto público para la atención de las necesidades de los adultos mayores se incrementa, aunado al estrechamiento de la PEA implicará un riesgo para el sostenimiento competitivo de Japón.

Para el año 2016, el 27.5\% de la población rebasaba los 65 años (mientras que la fuerza laboral descansaba en un rango de edad entre los 15 a los 60 años que representaba el 60.3\%), con apenas un $12.4 \%$ de niños hasta los 14 años. Se proyecta que para el 2050 el sector de la población en edad laboral se ubique en $51.8 \%$ y el de los niños disminuya a $10.6 \% .{ }^{19}$ Estos retos orillan a Japón a implementar una respuesta clara y el ejercicio de una eficiente política pública.

${ }^{17}$ Isabel Reynolds y Emi Nobuhiro. "As new era looms, women still face age-old challenges", The Japan Times, (31 de marzo de 2019). Disponible en: https://www. japantimes.co.jp/news/2019/03/31/national/social-issues/new-era-dawns-japanwomen-still-face-age-old-challenges/\#.XRFKWT9KgdV

${ }^{18}$ La tasa de natalidad que registra el número de nacimientos por cada mil habitantes de manera anual, para el 2018 fue del $7.4 \%$.

${ }^{19}$ Ministry of International Affairs and Communication. "Statistical Handbook of Japan”. Statistics Bureau (2017). Disponible en: https://www.stat.go.jp/english/data/ handbook/pdf/2017all.pdf 


\section{Del empleo y los jóvenes}

Los jóvenes japoneses viven en un dilema. Mientras algunos de ellos rechazan el camino laboral corporativo tradicional "seguro pero sofocante" que sus padres y abuelos aceptaron taciturnamente, otros optan por empleos de medio tiempo o por proyecto determinado que ofrecen más libertad, pero mucha menos seguridad.

La actual generación de japoneses está marcada por estereotipos. De manera recurrente se puede leer en los medios de comunicación o incluso escuchar a los adultos mayores, frases tales como: "los jóvenes de hoy no desean trabajar duro"; "los jóvenes no hablan corretamente y no tienen sentido común". ${ }^{20}$ Lo cierto es que la brecha generacional provoca estragos en todos los sentidos al propiciar tensión. Las personas mayores critican a los jóvenes por su egoísmo, rudesa e incapacidad para comunicarse, en realidad dicen que ellos carecen de los atributos necesarios para mantener las bases del sistema que sus antepasados se dedicaron a construir y mantener. ${ }^{21}$ De manera paradógica, es ese orden social agonizante y que desmitifica a un Japón igualitario, homogéneo y que las nuevas generaciones no desean conservar, por ofrecer limitadas alternativas para ellos.

En un Japón que se consideraba como una sociedad de clase media, el mal llamado "milagro económico" impactó en "todos" al crear, en efecto, mayores rangos de igualdad pero con la desigualdad invisibilizada. Jeff Kingston afirma que en 2000, la tasa de pobreza relativa excedió el $15 \%$, muy por encima del promedio de los miembros de la Organización para la Cooperación y el Desarrollo Económico (OCDE) que fue del 10\%; para 2016, aumentó a 16.3\%, lo que dejó una pesada carga en la sociedad japonesa si se considera el porcentaje de niños criados en pobreza relativa, que fue de casi $14 \%$, aproximadamente 3.5 millones de niños; de los cuales sólo 200,000 recibieron apoyo estatal, enton-

\footnotetext{
${ }^{20}$ Gordon Mathews y Bruce White. Japan's Changing Generations Are young people creating a new society? (London: Routledge Curzon, 2004), 14.

${ }^{21}$ Ibidem, pág. 15.
} 
ces se puede pensar hay una reproducción continua de las condiciones de desigualdad. ${ }^{22}$

Uno de los factores más relevantes para discutir la situación de los jóvenes es la condición laboral. La transición del modelo de empleo seguro y vitalicio a uno con mayor riesgo e inseguridad enfrenta a la sociedad con un dilema. Es un hecho que el cambio estructural de la economía japonesa fue impulsado a partir de la desregulación de los mercados laborales desde 1999. Las reformas neoliberales implementadas por el primer ministro Junichirō Koizumi (2001-2006) partieron de la afirmación de que el estilo de vida y el acceso al trabajo era una responsabilidad propia, lo que significaba que debían ser enfrentados por los propios ciudadanos. ${ }^{23}$ Los jóvenes se desenvuelven en este ambiente incierto. Aún con un buen salario, los trabajos de medio tiempo, por horas o por proyecto, en lo general, no les garantizan su futuro.

El ambiente precarizado se refleja aún más cuando las jóvenes japonesas se incorporan a la fuerza laboral, especialmente las casadas, que además de atender el hogar, deben buscar fuentes de ingresos adicionales para contribuir al ahorro y a los gastos familiares. Además, el empleo vitalicio favoreció una gran competencia entre los jóvenes que deseaban -al salir de la universidad-incorporarse a las grandes compañías reconocidas mundialmente. Así, obtener las mejores notas y concluir con éxito su preparación constituía una forma de vida y de reconocimiento social, también una forma de retribuir a la familia la inversión hecha en su persona. El sentido de reconocimiento, de pertenencia a un grupo y de realización se conviertían en factores que daban sentido a la vida de los jóvenes y a su compromiso por estudiar.

Las políticas públicas en Japón, si bien han intentado responder a esos retos, no han logrado cabalmente dar un sentido de seguridad a su sociedad. Por ejemplo, el objetivo de Japón, tal como se define en su estrategia de revitalización, es disminuir en $20 \%$ el desempleo en quienes no han conseguido un trabajo por más de seis meses, así como implementar políticas para atender la demanda laboral, mejorar la transición de la escuela al trabajo a través de hacer más eficiente la orientación profesional. Además, diseña 
un conjunto de estrategias dirigidas a las empresas y empleadores para mejorar los métodos de reclutamiento, provisión de capacitación y desarrollo profesional, orientando a jóvenes graduados, desempleados, trabajadores independientes y a NEETs. ${ }^{24}$

Otra de las políticas públicas que se han implementado, pero que no ha tenido el éxito suficiente para lograr incorporar plenamente a la juventud japonesa al escenario laboral es el plan de desafío de independencia juvenil ${ }^{25}$ que pretende crear una educación emprendedora participativa desde la primaria hasta la preparatoria, al favorecer una "academia de emprendedores" que aliente a los jóvenes a convertirse en empresarios. Esa medida ha sido considerada un eslabón para formar en los jóvenes con un carácter más independiente y acorde a la realidad del Japón de hoy.

De acuerdo a Tuukka Toivonen, ${ }^{26}$ el gobierno japonés enfrentó dificultades para incorporar a los jóvenes al campo laboral, ya que la casi ausencia de derechos existentes para ellos hacía imposible utilizar el apalancamiento para convertir los derechos en beneficios que están condicionados al empleo. La problemática, además, se complica, cuando muchos de los japoneses desemplados dependen del apoyo de sus familias, lo que suma nuevos actores al problema, si se considera que los padres pueden depender de la jubilación, tener un espacio pequeño para vivir o enfrentar los problemas propios de los adultos mayores. Las características del contexto japonés llevaron a los formuladores de política pública a desarrollar programas que se basen en lo que Toivonen llama "activación simbólica": políticas orientadas a la integración de los jóvenes en el mercado laboral.

Una tercera política pública que se ha impulsado para proporcionar mejores condiciones de incorporación al campo laboral es

\footnotetext{
${ }^{24}$ ILO. "Youth Employment Policy Summary JAPAN". International Labor Organization. Disponible en https://www.ilo.org/wcmsp5/groups/public/---asia/---robangkok/---sro-bangkok/documents/publication/wcms_534277.pdf

25 Tukka Toivonen. Japan's Emerging Youth Policy: Getting Young Adults Back to Work (London: Routledge, 2013), 45.
}

${ }^{26}$ Ibidem, pág. 45. 
el Plan de Medidas de Bienestar para Jóvenes Trabajadores, ${ }^{27}$ que incluye disposiciones para fomentar la juventud trabajadora a través de un sistema de mentores y asegurar tiempo por su autodesarrollo. Lo anterior busca que los empleadores se aseguren de que los jóvenes tengan tiempo para seguir estudiando, que estén mejor preparados y que ello les proporcione mejores opciones laborales.

No obstante, las políticas gubernamentales para el impulso laboral juvenil, la administración de Abe desde 2012, promovió que se crearan: el comité para la reforma regulatoria y el comité para la competitividad industrial, al abrir la posibilidad de que las empresas pudiesen despedir a sus empleados en función de su rendimiento, dando fin a una época de certeza en los jóvenes que estaban seguros que un buen desempeño universitario les permitiría acceder al sistema empleo vitalicio.

El ideal del esfuerzo recompensado con reconocimiento y éxito pierde sentido en una sociedad que trata de responder a las demandas externas con un modelo de organización social que no se ha adecuado, ni ha creado las condiciones para asumir los cambios en Japón. ${ }^{28}$ Los jóvenes japoneses acostumbrados a tener una situación laboral estable o bien atravesar sólo un corto periodo de tiempo desempleado, hoy no sólo es precario para los jóvenes, sino difícil para la sociedad en general. Las estadísticas oficiales incluyen en la categoría de empleado, sólo a quienes tienen un trabajo regular, mientras que quienes tienen una situación laboral de medio tiempo o temporal son ubicados en la categoria de "sin empleo" y "otros", generando confusión sobre lo que la política pública promueve y lo que mide, sin considerar además, lo que los jóvenes realmente esperan o desean. ${ }^{29}$

\footnotetext{
${ }^{27}$ ILO. "Japan, Basic Plan for Working Youth Welfare Measures". International Labor Organization. Disponible en: http://ilo.org/dyn/natlex/natlex4.detail?p_ lang=en\&p_isn=95611\&p_country=JPN\&p_count $=865$.

${ }^{28}$ María Elena Romero. "Hikikomori. Las voces silenciosas de la sociedad japonesa" México y la Cuenca del Pacifico 8, (23) (Mayo-agosto 2019), 123-138.

${ }^{29}$ Akio Inui. "Why Freeter and NEET are Misunderstood: Recognizing the New Precarious Conditions of Japanese Youth”, Social Work \& Society 3, no. 2 (2005), 244. Disponible en: https://www.socwork.net/sws/article/view/200/485
} 
Además, mientras en el mundo laboral ya no tiene las condiciones para atender la demanda de los jóvenes ávidos de logros. Lo anterior provoca situaciones sociales complejas, como desesperanza, la búsqueda de empleos por horas o tiempo parcial a cambio de un salario relativaente aceptable, lo que genera sentimientos de fracaso, depresión y constante incetidumbre ante la falta de certeza en el futuro.

Como se mencionó, el gobierno japonés aprobó una serie de reformas laborales diseñadas para resolver los retos demográficos y crear condiciones para promover el crecimiento del empleo, especialmente enfocándose a aquellos quienes desean encontrar un trabajo estable. ${ }^{30}$ Estas se concetraron en atender a un sector de 35 a 45 años quienes han tenido problemas en lograr una situación laboral estable, es decir a aquellos que concluyeron estudios universitarios entre los años 1993 y 2004.

De acuerdo al Banco Mundial, ${ }^{31}$ Japón tiene apenas un 2.9 \% de jóvenes que no están en el rubro de educación, empleo o capacitación (como porcentaje del total de la población juvenil en 2018). En cuanto al desempleo de personas con formación educativa, Japón ha logrado reducir la tasa, de $4.25 \%$ en el 2002 a 2\% para el 2018 (como porcentaje del total de la fuerza laboral con educación profesional)..$^{32}$ Esas cifras son relevantes indudablemente, pero quedan ligeramente eclipsadas frente a los niveles históricos de pleno empleo que logró tener durante su fase de alto crecimiento. Además, la legislación laboral al flexibilizar el empleo lo precarizó por lo que los egresados de las universidades

\footnotetext{
${ }^{30}$ Kyodo News Agency. "Cabinet OKs reforms to address Japan's labor market woes", The Japan Times (22 de junio de 2019). Disponible en: https://www. japantimes.co.jp/news/2019/06/22/business/economy-business/cabinet-oks-reforms-address-japans-labor-market-woes/?fbclid=IwAR2I-d2Sc2jDPSsU6o0o65VufIQjpOg2Lt123bDM88qibtc0ygAyAuWHcOo\#.XQ5tGOhKiUl)

31 WB. "Share of youth not in education, employment or training, total (\% of youth population)", World Bank, (2019). Disponible en: https://data.worldbank.org/indicator/SL.UEM.NEET.ZS

32 WB. "Unemployment with advanced education (\% of total labor force with advanced education)", World Bank (2019). Disponible en: https:/data.worldbank.org/ indicator/SL.UEM.ADVN.ZS.
} 
tienen que acceder a cualquier tipo trabajo y les obliga a vivir por más tiempo con sus padres.

Lo cierto es que en la nueva Era Reiwa, Japón enfrenta cambios sociales con efectos más severos en comunidades vulnerables siendo un reto que ha sido postergado. Por ejemplo, en un país como Japón dificilmente podría pensarse en marginados, aquellos que han quedado fuera del sistema y que no son públicamente reconocidos como problema social. Sin embargo, el recorrer ciertos distritos de Tokio se puede observar una realidad diferente: indigentes viviendo debajo de puentes o a las orillas del río, conocidos como homeless cuya existencia en los grandes centros urbanos japoneses ha destruido otros de los grandes mitos económicos del Japón contemporáneo como el paraíso de la clase media. ${ }^{33}$

Japon vive una etapa de contradicciones y expone un contexto para repensar la idea de la japoneidad como constructo de identidad cultural: ¿Qué significa ser japonés en la Era Reiwa? Repensar los valores versus los antivalores. Violencia, suicidios, aislamiento como disfunción social, son problemas que enfrentan los jóvenes, así como los sobrenombres de Freeter y NEETs, son una la realidad en la que la juventud japonesa espera encontrar un sentido de cohesión que les ofrezca una dirección a su vida.

Además del impacto que ejercen en la economía, los NEETs y los Freeter tienen un doble efecto negativo en la sociedad. Por un lado, la mayoría de esos jóvenes depende aún de sus padres, quienes tienen un subsidio limitado de gobierno, de manera que las condiciones de vida se deterioran rápidamente, desde la convivencia en espacios pequeños hasta el consumo de alimentos y el acceso a actividades recretativas se ve limitado. Por el otro lado, si esos jóvenes buscan tener una vida independiente de sus padres, la mayoría no tiene acceso a comodidades. ${ }^{34}$ De acuerdo con Hoang

\footnotetext{
33 Martha Loaiza, María Elena Romero y Cristina Tapia. "Juventud, pobreza y desesperanza en el Japón del siglo XXI”, Revista Miriada 8, no. 12 (2016). Disponible en: https://dialnet.unirioja.es/servlet/articulo?codigo $=5691918$.
}

${ }^{34}$ Linh Hoang. "Japanese NEET and Freeter: A representation of the relation- 
“muchos Freeter y Arubaito en Japón viven en Net Cafés ya que no pueden permitirse la compra o el alquiler de un apartamento; según una encuesta reciente del Gobierno japonés sobre las personas que tienen los denominados "Net Cafe Refugees", 5,400 personas pasaron al menos la mitad de la semana viviendo en cafés como Plaza Manga". ${ }^{35}$

Asimismo, algunos empleados de bajos ingresos, de medio tiempo o por horas a menudo viven en pequeños cubículos oscuros en cibercafés donde juegan videojuegos en su tiempo libre mientras ahorran para el alquile de una vivienda; conocidos como $<$ refugiados de cibercafés $>$, pasan todas las noches lavando, durmiendo y comiendo en esos centros recreativos entre lo que generalmente tienen un empleo mal pagado, esos jóvenes vivien en un mundo en el que van de un cubículo oscuro al siguiente mientras, se desempeñan en trabajos que los mantienen al borde de los niveles de pobreza relativa. ${ }^{36}$

De acuerdo con Andy Furlong, ${ }^{37}$ utilizar categorías como NEETs, Freeter, Arubaito para identificar a los jóvenes tiene sus ventajas y desventajas. La ventaja analítica es que permite ubicar mejor la situación de los jóvenes, al dejar de lado las categorías dicotómicas: empleado y desempleado, y hacerlos visibles frente a las políticas públicas para que atiendan las condiciones de precariedad de este sector. Sin embargo, la desventaja del término es la heterogeneidad de esos grupos y sus condiciones particulares.

ship between social changes and youth's employment pattern". Union College Union Honor Thesis, (2016), 54. Disponible en: https://pdfs.semanticscholar.org/ 50d7/009a16f7d743bde7148357d9ad5352872c6e.pdf.

35 Ibidem, pág. 55.

${ }^{36}$ Corey Charlton. "Japan's internet café refugees: The workers forced to move into tiny, dark cubicles because they can't afford to rent a flat", Daily Mail (1 de abril de 2015). Disponible en: https://www.dailymail.co.uk/news/peoplesdaily/article3021375/Japan-s-internet-caf-refugees-workers-forced-dark-tiny-cubicles-t-affordpay-rent.html

${ }^{37}$ Andy Furlong. "Not a very NEET solution: Representing problematic labor market transitions among early school leavers", Work, Employment and Society 20, no. 3 (September 2006), 553-569. Disponible en: doi:10.1177/0950017006067001. 
En suma, podría pensarse que la sociedad japonesa va cayendo en un círculo vicioso. Mientras los padres de jóvenes en los años setenta, ochenta, incluso a principios de los noventa del siglo XX invertían grandes cantidades de recursos en una preparación exhaustiva en sus hijos, al influir en ellos positivamente para aprobar exámenes rigurosos de las universidades más prestigiadas del país y asegurar con ello un empleo reconocido, en la actualidad, la realidad dice que menos jóvenes ingresan a las universidades deseadas, ya sea porque no tienen la suficiente preparación o no les interesa dedicar parte de su vida a estudiar cuando el egreso de esas instituciones de educación superior ya no les garantiza empleos bien remunerados.

\section{IV ¿Dreamers de Japón?}

Otro sector joven con condiciones precarias y que se encuentra en ese ambiente de invisibilidad, aunque parezca extraño, es el que podríamos denominar "Dreamers" que se ubican fuera y dentro Japón. Grupo que con características particulares, siendo quizá, lo único que comparten con sus similares en Estados Unidos, es que en el sentido literal son "soñadores".

En el caso japonés, pueden distinguirse dos categorías: la primera, se refiere a aquellos niños, niñas y jóvenes que fueron llevados por sus progenitores a Japón por cuestiones de trabajo o estudio en dicho país asiático, así pues, ingresaron a la escuela y aprendieron el idioma japonés donde se adaptaron (muchos de ellos con dificultad) a la cultura y a la sociedad. En la segunda categoría se ubican los nacidos en Japón y que no tienen derecho de obtener la nacionalidad por la normatividad jurídica basada en la jus sanguinis. En ambos casos, los hijos de inmigrantes tuvieron una experiencia de vida temporal en Japón ya que sus padres, ambos extranjeros, retornaron a sus países de origen o migraron a un tercero.

Por ejemplo, de acuerdo con datos del Ministerio de Asuntos Interiores y Comunicaciones, el número de residentes (de mediano y largo plazo) mexicanos en Japón en el 2015 fue de 2,141, se observó un ligero incremento en el siguiente año, al alcanzar 
2,304 y para el 2017 se contabilizaron $2,566 .^{38}$ Dentro de esos números, se ocultan los "Dreamers".

En la mayoría de los casos el retorno a los lugares de origen de sus padres fue complejo, sufrieron el "shock cultural de reserva" pero se adaptan, al final de cuentas, a la cultura de sus progenitores. Sin embargo, los que tienen oportunidad, continúan en contacto con Japón convirtiéndose, en realidad, en virtuales promotores de la japoneidad. Muchos de ellos desean regresar al país que les marcó parte de su infancia y juventud. Pero son imperceptibles en el diseño de las políticas públicas de Japón. El gobierno cuenta con programas específicos para la comunidad "nikkei" (descendientes de japoneses que viven ultramar) para el aprendizaje o perfeccionamiento en el idioma japonés, capacitación y otros más operados por la Agencia de Cooperación Internacional de Japón (JICA por sus siglas en inglés). No existe programa alguno para estos "Dreamers".

En el marco del acelerado envejecimiento en Japón y de su tasa de fertilidad (en el 2017 fue de 1.4 hijos o hijas por madre japonesa), se abre el debate sobre la necesidad de flexibilizar las políticas migratorias. En el 2016, se emitieron 257,739 visas para estudiantes extranjeros, algunos de ellos con la clara intención de continuar su formación académica y otros más con la idea de combinar o realizar trabajos de tiempo parcial. Asimismo, Tokio busca relajar los requisitos para el ingreso de extranjeros que puedan colocarse en espacios laborales definidos más allá de los orientados a la atención de los ancianos o de las actividades " $3 \mathrm{~K}$ ”, es decir, los oficios pesados, sucios y peligrosos que ya los jóvenes japoneses no desean ocupar. Se calcula que para el 2040, los japoneses mayores de 65 años representarán el $48 \%$ del total de la población; y se observará un decrecimiento a 107, 276 millones de los registrados en el 2016 que sumaron 126,193 millones. ${ }^{39}$

\footnotetext{
${ }^{38}$ Ministry of Internal Affairs and Communication. "Statistical Handbook of Japan”. Statistics Bureau (2019). Disponible en: https://www.stat.go.jp/english/data/ handbook/pdf/2019all.pdf

${ }^{39}$ David Green. “As Its Population Ages, Japan Quietly Turns to Immigration”, $M i$ gration Policy Institute (28 de marzo de 2017). Disponible en: https://www.migrationpolicy.org/article/its-population-ages-japan-quietly-turns-immigration
} 
Los "Dreamers" como una comunidad de personas con diferentes formaciones y habilidades profesionales, ante la caída de la tasa de fertilidad en ese país asiático, pueden contribuir a paliar el no muy lejano déficit de talentos y de mano de obra calificada en Japón. Su ventaja, es que ellos ya conocen a ese país asiático, hablan su idioma y conocen los códigos (escritos y no escritos) de la sociedad japonesa. Esos “soñadores” están en espera de una oportunidad para ser visibles en la Era Reiwa.

En suma, los "Dreamers" como los jóvenes japoneses, desean tener oportunidades laborales en medio de las políticas todavía restrictivas en materia migratoria de Japón, la apertura laboral es un tema que los grupos conservadores lo ligan al tema del mantenimiento de la "homogenidad racial", mientras otros observan la necesidad de atender el ya existente "déficit laboral" de talentos en Japón que por las propias contradicciones del sistema económico y social tampoco ha permitido un acceso fluído y constante para las nuevas generaciones de japoneses.

\section{Reflexiones Finales}

El nacimiento de la Era Reiwa, no tendrá un impacto directo en temas económicos o políticos. Sin embargo, tiene un significado psicológico y simbólico que permitirá que la sociedad se sienta cohesionada en torno a una figura que representa su cultura y tradición. Tal vez ello contribuya a enfrentar los cambios y retos que Japón continúa enfrentando en el siglo XXI.

En efecto, en 2019 inició la Era Reiwa pero el gobierno neoliberal continúa. La sociedad japonesa demanda respuestas reales a problemas cotidianos, especialmente en atender los problemas que heredaron, por lo que los jóvenes se ven obligados a cumplir con ciertas expectativas que el contexto no les permite obtener y ello genera frustación y desesperanza lo cual acrecenta los problemas sociales en Japón.

Dos acciones se visualizan como opciones y pueden dar respuesta a una juventud desesperanzada. La primera se refiere a la construcción de puentes que conecten al joven en formación con 
la realidad que enfrentan, que asuman el nuevo escenario como el reto que les toca paliar y reestructuren sus objetivos de vida. El ambiente que describe Haruki Murakami en la novela Tokio Blues de jóvenes que estudian en la universidad, pero dejan la casa familiar y son mantenidos por los padres; adolescentes que no tienen de que preocuparse más que esperar el apoyo de los padres y aprobar los exámenes, está terminado. La juventud japonesa se recompone y presiona por una recomposición social de su país. La segunda se enfoca a que es menester visibilizar los problemas y las diferentes demandas sociales que - dentro y fuera de Japón- emergen, como los de la comunidad de los "Dreamers" que puedan contribuir a las necesidades de empleo altamente calificado que requieran cada vez más las corporaciones japonesas en el mundo de los negocios caracterizado por una alta competencia.

\section{Fuentes}

Charlton, Corey. "Japan's internet café refugees: The workers forced to move into tiny, dark cubicles because they can't afford to rent a flat", Daily Mail, 1 de abril de 2015. https://www. dailymail.co.uk/news/peoplesdaily/article-3021375/Japans-internet-caf-refugees-workers-forced-dark-tiny-cubicles-tafford-pay-rent.html.

Furlong, Andy. "Not a very NEET solution: Representing problematic labour market transitions among early school leavers", Work, Employment and Society 20, no.3, September, 2006. doi:10.1177/0950017006067001.

Green, David. "As Its Population Ages, Japan Quietly Turns to Immigration", Migration Policy Institute, 28 de marzo de 2017. https://www.migrationpolicy.org/article/its-population-ages-japan-quietly-turns-immigration.

Hamaguchi, Keiichirō. "La problemática del modelo de empleo japonés". Nippon, 17 de septiembre de 2013. https://www.nippon.com/es/currents/d00088/.

Hoang, Linh. "Japanese NEET and Freeter: A representation of the relationship between social changes and youth' $s$ em- 
ployment pattern". Union College Union. Honor Thesis 2016. https://pdfs.semanticscholar.org/50d7/009a16f7d743bde7148357d9ad5352872c6e.pdf, 2016.

ILO. "Japan, Basic Plan for Working Youth Welfare Measures". International Labour Organization. http://ilo.org/dyn/natlex/natlex4.detail?p_lang=en\&p_isn=95611\&p_country=JPN\&p_count $=865$.

ILO. "Youth Employment Policy Summary JAPAN". International Labour Organization. https://www.ilo.org/wcmsp5/ groups/public/---asia/---ro-bangkok/---sro-bangkok/documents/publication/wcms_534277.pdf.

IMF. "World Economic Outlook Database”, International Monetary Forum, 2019. https://www.imf.org/external/pubs/ft/ weo/2019/01/weodata/index.aspx.

Inui, Akio. "Why Freeter and NEET are misunderstood: Recongnizing the New Precarious Conditions of Japanese Youth", Social Work \& Society 3, no. 2, 2005. http://www.socwork. net/Inui2005.pdf.

Japan Government. "ABENOMICS. For future growth, for future generations, and for a future Japan that is robust” (2017), https://www.japan.go.jp/abenomics/_userdata/abenomics/ pdf/170508_abenomics.pdf.

Japan Government. “The Constitution of Japan”. https://japan. kantei.go.jp/constitution_and_government_of_japan/constitution_e.html.

Kingston, Jeff. Critical Issues in Contemporary Japan. New York: Taylor and Francis, 2019.

Kingston, Jeff. Nationalism in Asia. A history since 1945. United States: Wiley Blackwell, 2017.

Kyodo News Agency. "Cabinet OKs reforms to address Japan's labor market woes", The Japan Times. 22 de junio de 2019. https://www. japantimes.co.jp/news/2019/06/22/business/economy-business/ cabinet-oks-reforms-address-japans-labor-market-woes/?fbclid=IwAR2I-d2Sc2jDPSsU6o0o65VufIQjpOg2Lt123bDM88qibtc0ygAyAuWHcOo\#.XQ5tGOhKiUl). 
Loaiza, Martha, María Elena Romero y Cristina Tapia. "Juventud, pobreza y desesperanza en el Japón del siglo XXI”, Revista Miriada 8, no 12, 2016. https://dialnet.unirioja.es/servlet/ articulo?codigo $=5691918$.

López Villafañe, Víctor y Carlos Uscanga (Coords.). Japón después de ser el número uno. Del alto crecimiento al rápido envejecimiento. Siglo XXI Editores, México, 2015.

Mathews, Gordon y White, Bruce. Japan's Changing Generations Are young people creating a new society? London: Routledge Curzon, 2004.

Ministry of International Affairs and Communication. "Statistical Handbook of Japan”. Statistics Bureau 2017. https://www. stat.go.jp/english/data/handbook/pdf/2017all.pdf.

Ministry of International Affairs and Communication. "Statistical Handbook of Japan”. Statistics Bureau 2019. https://www. stat.go.jp/english/data/handbook/pdf/2019all.pdf.

Murakami, Haruki. Tokio Blues. Editorial Tusquest, México, 2013.

Quartucci, Guillermo. "La edad del ensimismamiento". Conferencia Magistral Inaugural, ALADAA - México, Monterrey, Nuevo León 21-22 marzo de 2019.

Reynolds, Isabel y Emi Nobuhiro. "As new era looms, women still face age-old challenges", The Japan Times. 31 de marzo de 2019. https://www.japantimes.co.jp/news/2019/03/31/ national/social-issues/new-era-dawns-japan-women-still-faceage-old-challenges/\#.XRFKWT9KgdV.

Rodao, Florentino. "La Soledad del País Vulnerable", Crítica (2019): 9-17.

Romero, María Elena. "Hikikomori. Las voces silenciosas de la sociedad japonesa" México y la Cuenca del Pacífico 8, no. 23 (Mayo-Agosto 2019): 123-138.

Sieg, Linda. "Japan's Heisei era: Changes, growth and tragedies", Reuters 24 de abril de 2019. https://www.reuters.com/article/us-japan-emperor-era/japans-heisei-era-changes-growth-and-tragedies-idUSKCN1S00LM. 
Sugimoto, Yoshio. An Introduction to Japanese Society. London: Cambridge University Press, 2010.

Takahashi, Katsuya. "New Japanese era name Reiwa has lifted society: Suga”. Mainichi Shinbum. 1 de mayo de 2019. https://mainichi.jp/english/articles/20190501/p2a/00m/ 0na/023000c.

Takenaka, Keizo. "Meeting the Reiwa Era's challenges, diario de Japón", The Japan Times, 28 de abril de 2019. https://www. japantimes.co.jp/opinion/2019/04/28/commentary/japan-commentary/meeting-reiwa-eras-challenges/\#.XRE6Xz9KgdV.

"The transition from Heisei to Reiwa". Japan Times, 30 de abril de 2019. https://www.japantimes.co.jp/opinion/2019/04/30/ editorials/transition-heisei-reiwa/\#.XupIakVKiUl.

Toivonen, Tukka. Japan's Emerging Youth Policy: Getting Young Adults Back to Work. London: Routledge, 2013.

Uscanga, Carlos (Coord.). Japón y sus Alternativas de Desarrollo Económico hacia el Futuro. México: Seminario Universitario de Estudios Asiáticos-Universidad Nacional Autónoma de México, 2017.

WB. "Share of youth not in education, employment or training, total (\% of youth population)", World Bank, 2019. https://data.worldbank.org/indicator/SL.UEM.NEET.ZS.

WB. "Unemployment with advanced education (\% of total labor force with advanced education)”, World Bank, 2019. https:// data.worldbank.org/indicator/SL.UEM.ADVN.ZS. 